\title{
Bad things come easier to the mind but harder to the body: Evidence from brain oscillations
}

\author{
Christof Kuhbandner $^{1} \cdot$ Philipp Spachtholz $^{1} \cdot$ Bernhard Pastötter $^{1}$
}

Published online: 6 July 2016

(C) Psychonomic Society, Inc. 2016

\begin{abstract}
An intriguing finding of research on emotional processing is a discrepancy between perception and behavior. Perceptually, a robust finding is that negative stimuli are processed faster and more efficiently than positive stimuli. Behaviorally, a similarly robust finding is that response times are slower for negative than for positive stimuli. We proposed and tested a novel account to explain this still unexplained discrepancy, on the basis of the assumption that negative valence narrows perceptual processes to the benefit of speeded perception, but broadens motor processes at the cost of slowed responding. Participants performed a valence judgment task in which they responded with their left or right hand to negative and positive stimuli that were presented on the left or right, and we measured the activation of relevant/deactivation of irrelevant perceptual and motor processes, as revealed by the lateralization of electroencephalographic brain oscillations. Stimulus-related lateralization of alpha activity $(8-12 \mathrm{~Hz})$ over perceptual areas was increased for negative stimuli, indicating more efficient perceptual processing. By contrast, response-related lateralization of beta activity $(20-25 \mathrm{~Hz})$ over motor areas was decreased for negative stimuli, indicating less efficient response activation. Consistent with our predictions, more detailed analyses showed that both lateralization effects were caused by dynamics at the level of inhibiting irrelevant processes. For negative as compared to positive stimuli, the inhibition of irrelevant perceptual processes was increased, but the inhibition of irrelevant motor processes was decreased.
\end{abstract}

Christof Kuhbandner

christof.kuhbandner@ur.de

1 Department of Psychology, University of Regensburg, Universitätsstr. 31, 93053 Regensburg, Germany
These findings indicate that the discrepancy between perception and behavior in emotional processing may stem from asymmetrical effects of emotional valence on the breadth of cortical activations in perceptual and motor networks.

Keywords Emotion $\cdot$ Perception $\cdot$ Motor behavior $\cdot$ Response speed $\cdot$ EEG

\section{Introduction}

One widely examined characteristic of our psychological system is the responding to emotionally relevant stimuli. One intriguing finding revealed by numerous studies is a surprising discrepancy between perception and behavior. On the side of perception, the finding is robust that less stimulus input or exposure is required to detect a negative than to detect a positive stimulus, indicating that negative stimuli are processed faster and more efficiently than positive stimuli (e.g., Dijksterhuis \& Aarts, 2003; Kuhbandner, Spitzer, \& Pekrun, 2011; Nasrallah, Carmel, \& Lavie, 2009), a phenomenon that has been summarized by Baumeister and colleagues (Baumeister, Bratslavsky, Finkenauer, \& Vohs, 2001) in their comprehensive review on emotional processing in the oftcited quotation "bad is stronger than good." By contrast, on the side of behavior, a similarly robust finding is that when responses to emotional stimuli are examined with speeded response tasks, such as speeded naming, lexical decision, or valence judgment tasks, the response times for negative stimuli are slower than the response times for positive stimuli (Kuperman, Estes, Brysbaert, \& Warriner, 2014; Lepaennen, \& Hietanen, 2004; Unkelbach, von Hippel, Forgas, Robinson, Shakarchi, \& Hawkins, 2010), a phenomenon that has been assumed to be "so generally true that there aren't really any 
references for it" (Gordon Logan, quoted in Brendl, Markman, \& Messner, 2005, p. 354).

The faster and more efficient processing of negative stimuli is typically assumed to be adaptive because the cost of failure of appropriate responding can be much higher for negative than for positive stimuli (e.g., Baumeister et al., 2001). From such a point of view, however, it is difficult to explain why only stimulus perception is speeded, whereas responding is slowed. To explain this seemingly counterintuitive observation, an initial suggestion has been that the slower responding to negative stimuli may not reflect a true emotional effect, but rather an effect of nonemotional factors, such as differences in the frequency of occurrence, which had not been tightly controlled in previous studies (Kousta, Vinson, \& Vigliocco, 2009; Larsen, Mercer, \& Balota, 2006). However, more recent research has shown that the response speed advantage of positive over negative stimuli is still observed even when such factors are clearly ruled out (Kuperman et al., 2014; Unkelbach et al., 2010).

Another prominent account to explain the slowed responding to negative stimuli is based on emotion-induced attentional distraction. Specifically, it is assumed that when response time tasks are used that are actually unrelated to emotional contents (e.g., lexical decision tasks), the processing of negative stimuli diverts attention to the negativity of the stimulus, and thus delays responding on the emotionunrelated task (e.g., Estes \& Verges, 2008; Fox, Russo, Bowles, \& Dutton, 2001). However, such an account can only explain the slowed responding to negative stimuli in tasks in which the emotional content of these stimuli is unrelated to the to-be made response. When the emotional content is relevant for responding (e.g., in valence judgment tasks), diverting attention to the negativity of the stimuli should instead speed responses. An initial study by Estes and Verges (2008) seemed to support that a slowed responding for negative stimuli is only found in lexical decision tasks, but not in valence judgment tasks. However, a closer look at this study reveals that there was a possible confounding color effect, because all of the words were presented in red. Because red boosts performance for negative but not for positive words (Kuhbandner \& Pekrun, 2013; Moller, Elliot, \& Maier, 2009), color effects might have masked a speed advantage of positive over negative words. In fact, when color effects are adequately controlled, the typical response time advantage of positive over negative words occurs in valence judgment tasks, as well (e.g., Unkelbach, Fiedler, Bayer, Stegmüller, \& Danner, 2008).

To summarize, although the accounts above may at least partly explain the slowed responding to negative stimuli that has been observed in previous studies depending on the characteristics of the materials and response tasks used, the basic phenomenon that negative stimuli are perceived faster but responded to slower still remains to be fully explained. One common feature of the accounts above is that the slowed responding has been explained at the stage of stimulus perception. However, rather than originating at earlier stages of perception, it may be that the slowed responding to negative stimuli results from dynamics at the later stage of response preparation. Several lines of theory and research suggest that negative stimuli induce a more widespread activation of motor representations than do positive stimuli, which in turn may lead to a delay in behavioral responding because interference must be overcome in favor of one specific response option (e.g., Anderson, 1974).

A more widespread activation of motor representations by negative stimuli is suggested by evolutionary considerations. When encountering a stimulus, the variety of potentially functional behaviors is larger for negative than for positive stimuli. When encountering a positive stimulus, the general aim is to reach the reward associated with the stimulus, and this reward is typically reached by approach behavior (i.e., distance decrease), because other forms of behavior will not move one toward the stimulus. By contrast, when encountering a negative stimulus, the general aim is to avoid the negative consequences associated with the stimulus. This can be reached by a number of different behaviors, ranging from the absence of visible movement (hiding), to agitated motor outputs ranging from approach (i.e., distance decrease in order to fight) to withdrawal (i.e., distance increase in the interest of flight; e.g., Carretié, Albert, López-Martín, \& Tapia, 2009; Gray, 1987). Consistent with this view, the research on the link between emotional valence and behavioral responses has shown that whereas negative stimuli can facilitate both approach and withdrawal behavior, depending on contextual factors, positive stimuli only facilitate approach behavior (e.g., Krieglmeyer \& Deutsch, 2013; Kuhbandner, Vogel, \& Lichtenfeld, 2015; Paulus \& Wentura, 2016).

Finally, a more widespread activation of motor representations by negative stimuli is also supported by neurophysiological studies, showing that motor cortex excitability is generally increased during the perception of negative stimuli (Borgomaneri, Gazzola, \& Avenanti, 2014; de Oliveira et al., 2012). Although such findings have often been interpreted to reflect a facilitating effect of negative valence (e.g., Borgomaneri et al., 2014), they may instead reflect increased interference between the activated motor representations that delays responding. Although this is a post-hoc explanation, it seems rather plausible, given that an unspecific general increase in activity typically impairs the activation of a specific item because more task-irrelevant items exceed activation thresholds (e.g., Olivers \& Nieuwenhuis, 2006). In particular, given that no response times have been collected in the respective studies, it remains an open question whether the general increase in motor cortex excitability actually facilitated or impaired the execution of responses.

Although the possibility that the slowed responding to negative stimuli may result from dynamics at the motor level has 
sometimes been mentioned in previous studies (e.g., Estes \& Verges, 2008), to our knowledge, a more elaborate account has neither been theoretically formulated nor empirically tested. One reason for this caveat may be that distinguishing between emotional effects at the levels of stimulus perception and response activation is difficult with behavioral data alone, because the observable response performance represents the combined output of emotional effects on both stages. One possibility to study the processing pathways from stimulus presentation to response execution would be to examine the activation and deactivation of the neural populations associated with the different subprocesses of stimulus perception and response activation, as revealed by stimulus- and responserelated changes in ongoing oscillatory brain activities in the respective neural networks.

With regard to stimulus processing, there is strong evidence that stimulus-related changes of alpha activities $(\sim 10 \mathrm{~Hz})$ over posterior brain areas modulate the perception of stimuli by regulating cortical activation and deactivation in perceptual networks (e.g., Ergenoglu et al., 2004; Ray \& Cole, 1985). Specifically, it has been demonstrated that visuospatial processing of stimuli presented to the left or the right hemifield leads to a decrease of alpha power contralateral to the stimulus location, along with a concurrent increase of alpha power ipsilateral to the stimulus location, indicating the activation of relevant and the deactivation of irrelevant perceptual processes, respectively (i.e., sensory lateralization; see, e.g., Kelly, Lalor, Reilly, \& Foxe, 2006; Thut, Nietzel, Brandt, \& Pascual-Leone, 2006; Worden, Foxe, Wang, \& Simpson, 2000). With regard to response activation, there is strong evidence that response-related changes in beta activity $(\sim 20 \mathrm{~Hz})$ over motor areas modulate motor processes by regulating cortical activation and deactivation in motor networks (e.g., Joundi et al., 2012; Pogosyan, Gaynor, Eusebio, \& Brown, 2009). Specifically, it has been shown that response preparation involves a decrease of beta power that is typically more pronounced contralateral than ipsilateral to the responding hand, reflecting response activation or deactivation (i.e., response lateralization; e.g., Doyle, Yarrow, \& Brown, 2005; Kaiser, Birbaumer, \& Lutzenberger, 2001; Stancák \& Pfurtscheller, 1996).

The aim of the present study was to disentangle the emotional effects on stimulus processing and response activation by examining ongoing oscillatory brain activities during the responding to positive and negative stimuli. Although several previous electroencephalography (EEG) studies have already shown that neural activities in perceptual areas are enhanced for emotional as compared to nonemotional stimuli, as revealed by decreased alpha activity over parieto-occipital areas (De Cesarei \& Codispoti, 2011; Kuhbandner et al., 2009), the effects of the emotional significance of stimuli on activation and deactivation in motor neural networks have, to our knowledge, not been examined to date. To examine this issue, we recorded the EEG signals of participants while they performed a valence judgment task in which they categorized positive and negative words. To examine the effect of emotional valence on perceptual networks, we measured alpha activity at recording sites over the visual cortex time-locked to stimulus onset; to examine the effect on motor networks, we measured beta activity at central recording sites over the motor cortex time-locked to the overt response. To examine sensorylateralization effects in alpha activity and responselateralization effects in beta activity, stimuli were presented to either the left or right of fixation, and participants responded to the valence of stimuli with either left- or right-hand responses (see Fig. 1A).

Behaviorally, we expected to replicate the finding that response times are slower for negative than for positive stimuli. At the electrophysiological level, we expected to replicate the finding that ongoing oscillatory activity in the alpha frequency range is modulated by the emotional valence of stimuli. Specifically, we expected to find larger sensory lateralization in stimulus-locked alpha power for negative than for positive stimuli, indicating more efficient processing of negative stimuli in perceptual networks due to the stronger activation of relevant (i.e., perceptual activities in the half-field where the stimulus was presented) and the weaker activation of irrelevant (i.e., perceptual activities in the half-field where the stimulus was not presented) perceptual activities. Most importantly, we expected to find smaller response lateralization in response-locked beta power for negative than for positive stimuli. Such a finding would demonstrate that, despite the more efficient perceptual processing, response preparation in motor networks is less efficient for negative stimuli due to weaker activation of the relevant (i.e., motor activity associated with the responding hand) and stronger activation of the irrelevant (i.e., motor activity associated with the nonresponding hand) motor activities.

\section{Method}

\section{Participants}

On the basis of previous studies showing that robust response lateralization effects in beta activity were found with 12 participants (Doyle et al., 2005; Stancák \& Pfurtscheller, 1996), since we were manipulating the additional factor of Valence, we planned to include data from at least 24 participants. Twenty-eight right-handed undergraduate students ( $21 \mathrm{fe}$ males and seven males) participated for course credit after giving informed consent. The mean age of the participants was 25.5 years $(S D=10.4)$. Two of the participants were excluded from the analyses because they had massive muscle artifacts in their EEG. 
a

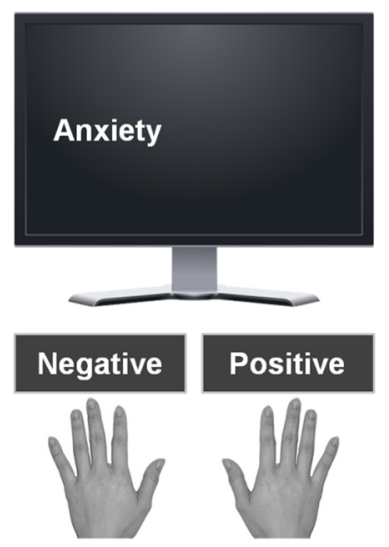

Fig. 1 Experimental procedure and behavioral results. (A) Participants performed a valence judgment task in which they categorized emotionally positive or negative words, presented at left and right screen positions, as positive or negative with either left- or right-hand responses. (B)

\section{Materials}

A total of 25 positive and 25 negative five- to six-letter German nouns were drawn from the Berlin Affective Word List-Reloaded (BAWL-R; Võ et al., 2009), the Leipzig Affective Norms for German (Kanske \& Kotz, 2010), and a large-scale database of affective norms (Lahl, Göritz, Pietrowsky, \& Rosenberg, 2009). The subsets of positive and negative nouns did not differ in the extremity of their valence $\left(M_{\text {neg }}=-1.96, S D=0.29 ; M_{\text {pos }}=2.10, S D=0.33\right.$; range: -3 to 3$)$, arousal $\left(M_{\text {neg }}=3.61, S D=0.51 ; M_{\text {pos }}=3.42\right.$, $S D=0.49$; range: 1 to 5$)$, frequency of usage $\left(M_{\text {neg }}=29.1, S D\right.$ $\left.=34.8 ; M_{\text {pos }}=29.0, S D=35.0\right)$, and number of letters $\left(M_{\text {neg }}=\right.$ $\left.5.64, S D=0.49 ; M_{\text {pos }}=5.52, S D=0.51\right)$, with $p \mathrm{~s}>.1410$.

\section{Design and procedure}

All manipulations and measures are reported. The stimuli were presented on a white background using E-Prime software version 2.0 (Psychology Software Tools, Inc., Pittsburgh, PA) with a viewing distance of about $120 \mathrm{~cm}$. The positive and negative words were presented within two blocks of 100 trials each. Within each block, each word was presented two times, once at the left and once at the right side of the screen; the trial order was randomized. Participants were instructed to categorize each word as quickly and accurately as possible as either "negative" or "positive" by pressing a left or a right response key on a gaming keypad (Hama, Monheim, Germany). The assignment of emotional categories to response buttons was counterbalanced across the two blocks within participants. Each trial started with a prestimulus interval with a random duration of 1,250-1,450 ms, showing a fixation point in the center of the screen. In the stimulus interval, the fixation point was removed and a word (letter size: $0.43^{\circ}$ ) was presented with the center of the word shifted either $10.8^{\circ}$ to either the

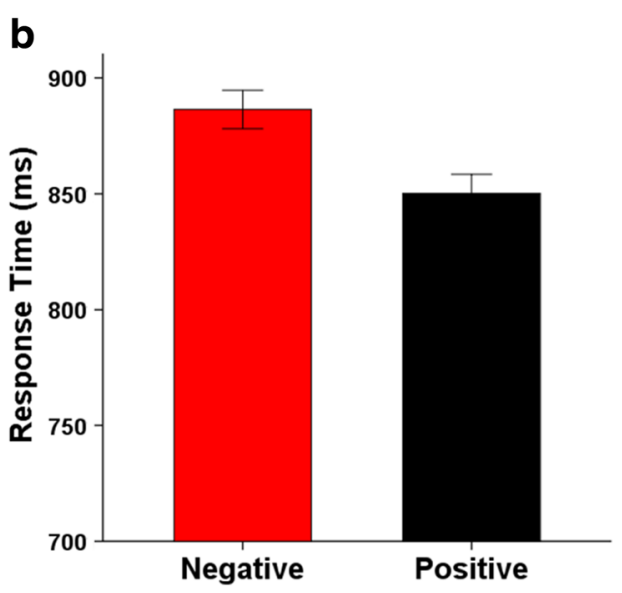

Response time performance as a function of the emotional valence of the words (negative, positive; $N=26$ ). Error bars represent withinsubjects $95 \%$ confidence intervals (Cousineau, 2005)

left or the right of fixation. Each trial ended with the participant's response, at which point the word disappeared, and the next trial started.

\section{EEG recording and analyses}

EEG was recorded from 21 active electrodes positioned on the scalp according to the extended 10-20 system, and two additional electrodes A1 and A2 were connected to the left and right mastoids, respectively. The EEG signals were originally recorded against a reference electrode placed at $\mathrm{FCz}$ and later re-referenced against a common average reference. A vertical electrooculogram (EOG) was recorded from one additional channel below the left eye, to control for eye movements and blinks. Signals were amplified between 0.016 and $250 \mathrm{~Hz}$ and were digitized with a sampling rate of $500 \mathrm{~Hz}$ (BrainAmp Amplifieres, Brain Products, Gilching, Germany). The impedances of all electrodes were kept below $20 \mathrm{k} \Omega$.

Prior to the data analysis, the EEG data were corrected for EOG artifacts by using calibration data and generating individual EOG artifact coefficients (Ille, Berg, \& Scherg, 2002), as implemented in the BESA Research software package (version 6.0; BESA Software, Gräfelfing, Germany). Any remaining artifacts were marked by careful visual inspection. The mean numbers of artifact-free segments were $84.1(S D=$ 8.21; range from 69 to 99) for negative words, and 84.2 ( $S D$ $=7.78$; range from 69 to 99 ) for positive words.

The EEG data were transformed into the time-frequency domain using a complex demodulation algorithm as implemented in BESA (see Hoechstetter et al., 2004). The algorithm consists of a multiplication of the time-domain signal with a complex periodic exponential function having a frequency equal to the frequency under analysis, and subsequent low-pass filtering. The data were filtered to a frequency range from 2 to $30 \mathrm{~Hz}$. The time resolution was set to $78.8 \mathrm{~ms}$ (full 
power width at half maximum, FWHM), and the frequency resolution was set to $1.42 \mathrm{~Hz}$ (FWHM). The time-frequency data were exported in bins of $50 \mathrm{~ms}$ and $1 \mathrm{~Hz}$.

Both stimulus-related and response-related changes in oscillatory brain activity were calculated. Stimulus-related changes were calculated from -200 to $1,000 \mathrm{~ms}$ time-locked to stimulus onset; response-related changes were calculated from $-1,000$ to $200 \mathrm{~ms}$ time-locked to response onset. To avoid filter artifacts at the edges of these time windows, all analyses were based on EEG segments ranging from $-2,000$ to $2,000 \mathrm{~ms}$ around the stimulus and response onsets, respectively. Stimulus-related and response-related power changes were determined by calculating the temporal-spectral evolutionthat is, the stimulus- and response-related power changes for all time-frequency points with power increases or decreases at time point $t$ and frequency $f$ related to the mean power at frequency $f$ over a preceding baseline interval (see Pfurtscheller \& Aranibar, 1977). Stimulus-related power changes were determined in relation to a prestimulus baseline interval that was set from -200 to $0 \mathrm{~ms}$ time-locked to stimulus onset, whereas response-related power changes were determined in relation to a preresponse baseline interval that was set from $-1,000$ to $-800 \mathrm{~ms}$ time-locked to response onset. Note, however, that the results and conclusions were the same when we used the same 200-ms prestimulus baseline interval for both stimulus-related and response-related analyses.

To examine sensory lateralization in the perception networks, averaged stimulus-induced power changes were calculated, separately for a left pool (P7, P3, O1) and a right pool $(\mathrm{P} 8, \mathrm{P} 4, \mathrm{O} 2)$ of posterior electrodes, based on the average-referenced data (e.g., Kelly et al., 2006; Thut et al., 2006). Using these pools of electrodes, sensorylateralization indices were calculated by subtracting the stimulus-induced power changes ipsilateral to the stimulus location from the power changes contralateral to the stimulus location, averaged across left and right stimulus locations, separately for positive and negative stimuli. Time-frequency spectrograms of significant differences in sensory-lateralization indices over the left and right posterior electrode pools between positive and negative stimuli were created by calculating $t$ tests for all timefrequency points $(725=25$ [time bins] $* 29$ [frequency bins]). A cluster analysis was calculated to determine the significant time-frequency windows of differences in sensory lateralization by using BESA Statistics (version 1.0; BESA Software, Gräfelfing, Germany). In cluster analysis, only adjacent time-frequency points that fell below a $p$ value of .05 in the $t$ test were considered. For each cluster, the sum of the $t$ values of the single significant time-frequency points of the empirically derived timefrequency cluster was calculated as a test statistic. Next, for each cluster, 5,000 random permutations were run in which the sum test statistic was repeated for randomly shuffled data sets, with the data randomly reordered across valence conditions, and the cluster with the highest sum of $t$ values was kept. By these means, null distributions were created from random permutation runs, and the critical $p$ values for the empirically derived time-frequency clusters were calculated. Only clusters with a critical $p$ value below .05 were analyzed further.

To examine response lateralization in motor networks, the response-related power changes at central electrodes $\mathrm{C} 3$ and C4 were calculated (e.g., Pfurtscheller, Brunner, Schlögl, \& Lopes da Silva, 1999; Stancák \& Pfurtscheller, 1996). Response-lateralization indices were calculated by subtracting the response-related power changes ipsilateral to the responding hand from the power changes contralateral to the responding hand, averaged across left- and right-hand responses, separately for negative and positive stimuli. Timefrequency spectrograms of significant differences in the response-lateralization indices over central electrodes and a cluster analysis of the differences in response-lateralization indices were determined and calculated analogously to the stimulus-related analysis steps above.

\section{Results}

\section{Behavioral results}

Accuracy was high and did not vary as a function of valence $\left(M_{\mathrm{Neg}}=.962, S D=.035 ; M_{\mathrm{Pos}}=.973, S D=.024\right), t(25)=-$ $1.64, p=.115, d=0.32,95 \%$ CI: -.023 to .003 . For the response time analysis, following typical conventions (see Balota \& Yap, 2011, for a review), errors (3.4 \% of the trials), and response times $2.5 S D$ s above or below each participant's mean response time ( $2.4 \%$ of the trials) were removed. Response time results are shown in Fig. 1B. Response times were slower for negative than for positive stimuli $\left(M_{\mathrm{Neg}}=\right.$ $\left.886 \mathrm{~ms}, S D=148 \mathrm{~ms} ; M_{\text {Pos }}=850 \mathrm{~ms}, S D=132\right), t(25)=$ $4.54, p<.001, d=0.89,95 \%$ CI: $20-53 .{ }^{1}$

\section{EEG results}

Sensory lateralization was determined by subtracting the stimulus-induced power changes ipsilateral to the stimulus location from the power changes contralateral to the stimulus location (e.g., Kelly et al., 2006; Thut et al., 2006), and response lateralization was determined by subtracting the response-related power changes ipsilateral to the responding

\footnotetext{
${ }^{1}$ To rule out the possibility that the observed results might have been driven by a few items, the data were also analyzed by collapsing over subjects and treating items as a random effect. The conservative $\operatorname{minF}^{\prime}$ statistic (Clark, 1973) indicated that the results were generalizable over both subjects and items, $\min F^{\prime}(1,70)=5.15, p=.026$.
} 
hand from the power changes contralateral to the responding hand (e.g., Pfurtscheller et al., 1999; Stancák \& Pfurtscheller, 1996). Figure 2A shows time-frequency spectrograms of the significant differences between positive and negative words in sensory lateralization, time-locked to stimulus onset (upper panel), and in response lateralization, time-locked to the response (lower panel).

Cluster analysis showed that differences between negative and positive words in stimulus-induced alpha lateralization over perceptual networks were most evident from 8 to $12 \mathrm{~Hz}$ and from 200 to $400 \mathrm{~ms}$ following stimulus onset, whereas differences in response-related beta lateralization over motor networks were most evident from 20 to $25 \mathrm{~Hz}$ and from -500 to $-100 \mathrm{~ms}$ before response onset. Figure $2 \mathrm{~B}$ shows time courses of the sensory-lateralization effect in posterior alpha power (upper panel) and the response-lateralization effect in central beta power (lower panel) as a function of emotional word type. Differences between negative and positive words in alpha activity occurred relatively early after stimulus onset, whereas differences in beta activity occurred relatively long before response execution. Figure $2 \mathrm{C}$ shows topographical maps and difference maps of the stimulus-induced changes in alpha power and the response-related changes in beta power as a function of emotional word type. This demonstrates that the stimulus-related differences in alpha power were most prominent over posterior electrodes, whereas the response-related differences in beta power were most prominent over $\mathrm{C} 3$ and $\mathrm{C} 4$. $^{2}$

Likewise, a $t$ test on the averaged difference in sensory-lateralization indices between negative and positive words, with differences averaged across the time-frequency points of the significant alpha cluster, indicated a significant effect of word type, $t(25)=-3.25, p=.003, d$ $=0.63,95 \%$ CI: $-17.6 \%$ to $-3.9 \%$. The averaged sensory lateralization in alpha activity was more pronounced for negative than for positive words ( $-25.2 \%$ vs. $-14.4 \%)$, indicating more efficient processing of negative than of positive stimuli in perceptual neural networks. A $t$ test on the averaged difference in response-lateralization indices between negative and positive stimuli, with differences averaged across time-frequency points of the significant beta cluster, showed a significant effect of word type, $t(25)=2.99, p=.006, d=0.59,95 \% \mathrm{CI}: 2.8 \%$ to $15.0 \%$. The averaged response lateralization in beta activity was less pronounced for negative than for positive

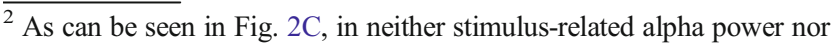
response-related beta power was a reliable effect observed over frontal electrode sites. Because eye movements should project mainly to frontal sites, it is unlikely that horizontal eye movements may have contributed to the present effects.
}

words $(1.3 \%$ vs. $-7.6 \%)$, indicating less efficient response activation in motor neural networks when responding to negative than to positive stimuli. To further examine the relationship between lateralization in alpha and beta activities and response times, we computed within-subject correlations (Bland \& Altman, 1995) between the respective lateralization indices and response times. There was a significant positive relationship between the lateralization of response-locked beta activity and response time, $r=.39, p=.047$, indicating that the speed of responding decreased with decreased response lateralization in beta power (note that the lateralization index is inversely related to the amount of lateralization). By contrast, lateralization of stimulus-locked alpha power was not correlated with response time, $r=-.23$, $p=.256$, indicating that sensory lateralization in alpha activity was unrelated to the speed of responding. ${ }^{3}$

Basically, the observed differences in lateralization may originate from differential effects on neural activity in brain areas contralateral or ipsilateral to stimulus location (alpha) and response location (beta), or both. Figure 3 shows the stimulus-related changes in alpha power (A) and response-related changes in beta power (B) for negative and positive words, separately for contralateral and ipsilateral recording sites.

To analyze whether the effects of emotion differed between contralateral and ipsilateral sites, we conducted analyses of variance (ANOVAs) with the factors Emotional Valence (negative vs. positive) and Recording Site (contralateral vs. ipsilateral). For both stimulus-related changes in alpha power and responserelated changes in beta power, the results showed a significant interaction between emotional valence and recording site, $F(1,25)=10.56, p=.003, \eta_{\mathrm{p}}{ }^{2}=.30$, and $F(1,25)=8.95, p=.006, \eta_{\mathrm{p}}{ }^{2}=.26$, indicating that the effects of emotional valence varied depending on laterality. Analyzing the effects of emotional valence separately for contralateral and ipsilateral recording sites showed that the activity at contralateral sites did not differ significantly between negative and positive words,

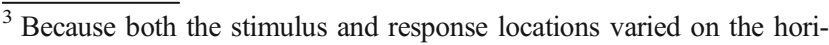
zontal dimension, it could be argued that stimulus-response compatibility effects may have influenced the present results. In fact, research on the spatial Simon effect has demonstrated that left-hand responses can be faster and less error-prone to stimuli presented on the left side than to stimuli presented on the right side of fixation, whereas the reverse is true for right-hand responses (see Simon, 1990). We therefore calculated additional analyses in which we examined the possible effects of spatial congruency on measures of response time and EEG alpha and beta oscillations. The results showed no stimulus-response compatibility effect and no interaction of the valence of stimuli with stimulus-response compatibility (all $p \mathrm{~s}>.10$ ), indicating that stimulus-response compatibility did not influence the present response time and EEG results.
} 


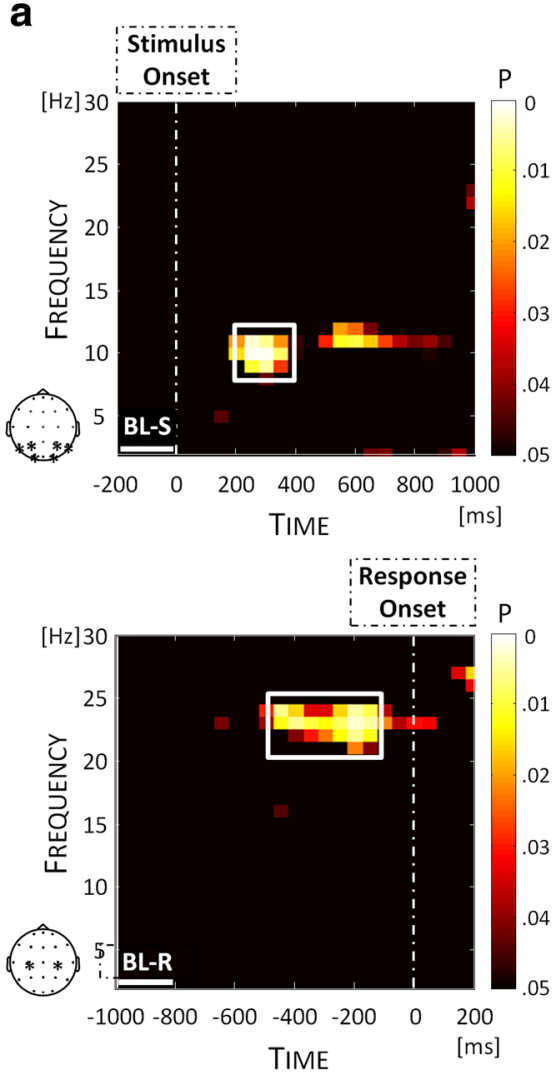

Fig. 2 EEG results. (A) Time-frequency spectrograms of significant differences in stimulus-related lateralization (upper panel) and responserelated lateralization (lower panel) of EEG power changes between positive and negative stimuli (see the Method section for an exact calculation of lateralization indices). In the calculation of stimulusrelated lateralization, power changes were time-locked to stimulus onset and calculated relative to a prestimulus baseline interval (BL-S); for the calculation of response-related lateralization, power changes were timelocked to response onset and calculated relative to a preresponse baseline interval (BL-R). Cluster analyses revealed a significant sensory lateralization effect in alpha power $(8-12 \mathrm{~Hz}$ ) from 200 to $400 \mathrm{~ms}$, time-locked to stimulus onset, and a response lateralization effect in

for both alpha power $(-13.8 \%$ vs. $-11.8 \%), t(25)=-$ $1.05, p=.302, d=0.14,95 \% \mathrm{CI}:-6.0 \%$ to $1.9 \%$, and beta power $(-24.4 \%$ vs. $-27.7 \%), t(25)=1.23, p$ $=.206, d=0.25,95 \% \mathrm{CI}:-1.9 \%$ to $8.4 \%$. By contrast, activity at ipsilateral sites did vary as a function of emotional valence, for both alpha power $(11.4 \%$ vs. $2.6 \%), t(25)=2.92, p=.007, d=0.57,95 \% \mathrm{CI}$ : $2.6 \%$ to $14.9 \%$, and beta power $(-25.7 \%$ vs. $20.0 \%), t(25)=-2.51, p=.019, d=0.49,95 \% \mathrm{CI}$ : $-10.3 \%$ to $-1.0 \%$. Indeed, whereas sensory lateralization in alpha activity was present for both positive words, $t(25)=-3.42, p=.002, d=0.67,95 \%$ CI: $23.1 \%$ to $-5.7 \%$, and negative words, $t(25)=-5.59, p$ $<.001, d=1.10,95 \%$ CI: $-34.4 \%$ to $-15.9 \%$, response lateralization in beta activity was present for positive words, $t(25)=-3.31, p=.003, d=0.65$, $95 \%$ CI: $-12.4 \%$ to $-2.9 \%$, but was absent for
C

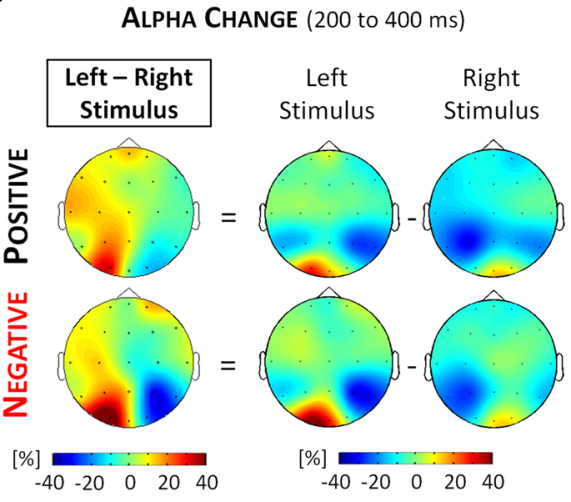

Beta Change ( -500 to $-100 \mathrm{~ms}$ )

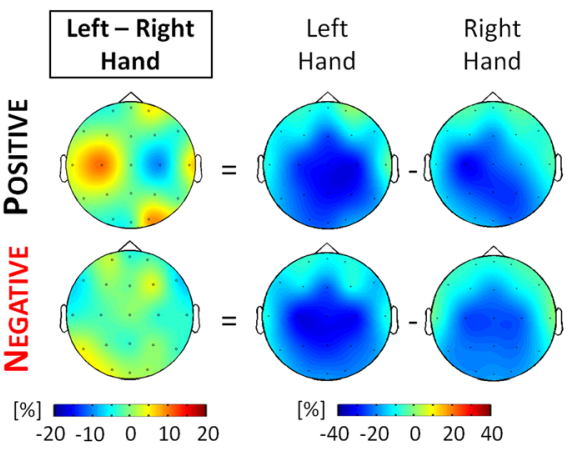

beta power $(20-25 \mathrm{~Hz})$ from -500 to $-100 \mathrm{~ms}$, time-locked to response onset. (B) Time courses of stimulus-related alpha lateralization over posterior electrodes (upper panel) and response-locked beta lateralization over central electrodes (lower panel) as a function of emotional word type. (C) Topographical maps and difference maps of stimulus-induced alpha power changes, with right-stimulus-induced alpha changes subtracted from left-stimulus-induced alpha changes (upper panel), and response-locked beta power changes, with right-hand response-locked beta changes subtracted from left-hand response-locked beta changes (lower panel). Topographical maps show that the effects in stimulus-related alpha power and response-related beta power were restricted to posterior and central areas, respectively

negative words, $t(25)=-0.68, p=.500, d=0.13$, $95 \%$ CI: $-5.1 \%$ to $2.5 \%$.

Finally, to examine whether the observed effects varied as a function of the hemifield of presentation or responding hand, ANOVAs were computed with the factors Emotional Valence (negative vs. positive) and Hemifield of Presentation (left vs. right) for sensoryrelated alpha lateralization, or Emotional Valence and Responding Hand (left vs. right) for response-related beta lateralization. For alpha lateralization, the analysis revealed neither a main effect of hemifield, $F(1,25)<$ $1, p=.375$, nor a valence-by-hemifield interaction, $F(1$, $25)<1, p=.652$, indicating that the effects of emotional valence on sensory lateralization in alpha activity did not vary as a function of hemifield of presentation. For beta lateralization, we observed a significant main effect of responding hand, $F(1,25)=4.45, p=.045$, 
a

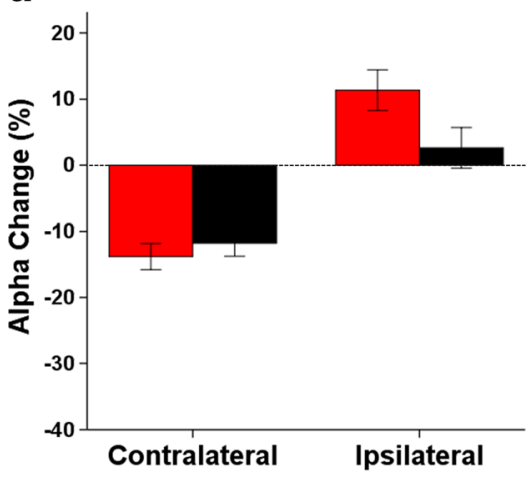

Fig. 3 Emotional effects at contralateral and ipsilateral recording sites. (A) Stimulus-related changes in alpha power and (B) response-related changes in beta power for negative and positive words are shown, separately for contralateral and ipsilateral recording sites. The results indicate that the effects of emotions on lateralization in both perceptual

$\eta_{\mathrm{p}}^{2}=.15$. Response lateralization in beta activity was more pronounced for right-hand than for left-hand responses $(-8.4 \%$ vs. $2.0 \%)$, reflecting an effect of hand dominance. However, we found no valence-byresponding-hand interaction, $F(1,25)=1.37, p=.253$, indicating that the effect of emotional valence on response lateralization in beta activity did not vary as a function of responding hand. ${ }^{4}$

\section{Discussion}

By examining the activations and deactivations of neural populations associated with stimulus perception and response preparation, in the present study we demonstrated an asymmetry in the processing of positive and negative

\footnotetext{
${ }^{4}$ In addition to EEG alpha and beta brain oscillations, event-related potentials (ERPs) have been related to perceptual and motor processes in prior EEG work (e.g., Hillyard \& Anllo-Vento, 1998; Leuthold, Sommer, \& Ulrich, 1996), and both perceptual and motor-related ERPs have been shown to be modulated by the emotional valence of stimuli (Eder, Leuthold, Rothermund, \& Schweinberger, 2012; Kissler, Herbert, Peyk, \& Junghöfer, 2007). In contrast to ERPs, which capture only phaselocked (evoked) activity, however, brain oscillations capture both phase-locked and non-phase-locked (induced) activities. Brain oscillations therefore may provide more sensitive markers of cortical activation and deactivation in perceptual and motor networks, a view that is supported by EEG work comparing perception- and motor-related effects on ERPs and alpha/beta oscillations (de Jong, Gladwin, \& Hart, 2006; Green $\&$ McDonald, 2010). Nevertheless, as a complementary analysis, we additionally examined stimulus- and response-locked lateralized readiness potentials (S-LRP and LRP-R; for theoretical and methodological details, see, e.g., Eder et al., 2012). Consistent with the present results, the analyses showed a larger LRP-R amplitude for positive than for negative words, $F_{\mathrm{c}}(1,25)=12.76, p=.001$ (peak interval of $50 \mathrm{~ms}$; for details, see Ulrich \& Miller, 2001), but no difference in LRP-R onsets, $p=.784$. Because of large variances in response times across both subjects and items, no reliable determination of S-LRP amplitude or onset was possible after visual inspection of the averaged signals.
}

\section{b}

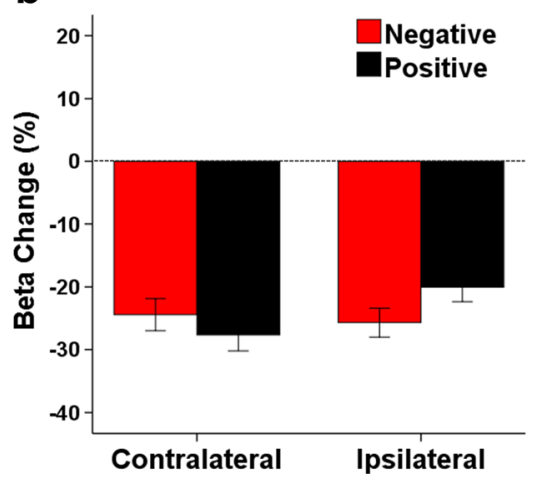

and motor areas originated from effects on neural activity in areas ipsilateral to the stimulus location (alpha) or to the response location (beta). Error bars represent within-subjects $95 \%$ confidence intervals (Cousineau, 2005)

stimuli at the level of cortical activations. Sensory lateralization in alpha activity over perceptual areas was more pronounced for negative than for positive words, indicating that negative stimuli were processed more efficiently in perceptual networks. By contrast, response lateralization in beta activity over central areas was more pronounced for positive than for negative words, indicating that response preparation in motor networks was less efficient for negative stimuli.

Further analyses showed that differential effects of emotional valence on both stimulus-related alpha activity and response-related beta activity were observed only at recording sites ipsilateral to the stimulus location (alpha) and the response location (beta), whereas at contralateral sites similar decreases in alpha and beta activities were observed for both types of emotional stimuli. Because the stimuli were presented on either the left or the right, and because participants responded with either the left or the right hand, contralateral decreases of alpha and beta activities reflect the processing of task-relevant information, and ipsilateral increases reflect the inhibition of taskirrelevant activity (Doyle et al., 2005; Kelly et al., 2006; Stancák \& Pfurtscheller, 1996; Thut et al., 2006). Accordingly, emotional valence seems to differentially influence only the (de)activation of irrelevant, but not the activation of relevant, stimulus- and response-related representations. Specifically, regarding perception, negative valence seems to lead to a stronger inhibition of irrelevant perceptual information, a finding that is consistent with behavioral findings showing enhanced processing of negative information at the cost of peripheral information (Christianson, \& Loftus, 1991). By contrast, regarding response preparation, negative valence seems to lead to weaker inhibition of irrelevant motor activity, a finding that is in line with recent findings showing that motor cortex excitability is generally increased during the 
perception of negative stimuli (Borgomaneri et al., 2014; de Oliveira et al., 2012)..$^{5}$ Thus, in other words, negative stimuli seem to narrow perceptual but broaden motor processes.

Such an asymmetrical effect on activations of the perceptual and motor networks may explain the surprising behavioral finding that, although negative stimuli are perceived faster than positive stimuli (e.g., Dijksterhuis \& Aarts, 2003; Nasrallah et al., 2009), response times are slower for negative than for positive stimuli (e.g., Kuperman et al., 2014; Lepaennen, \& Hietanen, 2004). At the level of perception, increased activation of relevant and deactivation of irrelevant perceptual information when processing negative stimuli may allow quick identification of such stimuli. However, despite the more efficient processing of negative stimuli, the increased activation of alternative motor representations during response preparation may bring about a delay in the responding to such stimuli due to increased interference.

From an evolutionary point of view, such an asymmetry in cortical activities between positive and negative stimuli may be adaptive. Regarding perception, the evolutionary pressure to quickly identify environmental stimuli may have been higher for negative than for positive stimuli because the costs of missing an environmental stimulus can be higher for negative than for positive stimuli (Baumeister et al., 2001). By contrast, regarding response preparation, a larger variety of activated motor representations may have the advantage of allowing for more flexible responding. Since the variety of potentially functional behaviors is larger for negative than for positive stimuli (Krieglmeyer \& Deutsch, 2013; Kuhbandner et al., 2015), the benefits of an increased activation of alternative motor representations may be especially large for negative stimuli, and outweigh the costs of delayed responding.

Whereas the account above suggests that the slowed responding to negative stimuli results from dynamics at the later stage of response preparation, some authors have suggested that the delay in the responses to negative stimuli may emerge at the earlier stage of cognitive stimulus analysis. For instance, on the basis of the assumption that positive information is more similar to other positive information, in comparison with the similarity of negative information to other

\footnotetext{
${ }_{5}^{5}$ Following previous work on alpha/beta event-related desynchronization (ERD) and synchronization (ERS), we interpret ERD as indexing the activation of relevant stimulus or response representations, and ERS as indexing the deactivation or inhibition of irrelevant stimulus or response representations. This view is based on previous evidence from work using, for instance, EEG, magnetoencephalography, transcranial magnetic stimulation, and electromyography in various attention and motor tasks, linking alpha/beta ERD to activation of relevant, and ERS to deactivation or inhibition of irrelevant, representations (see the "introduction" section). However, with the present results, we cannot rule out that other processes, including processes that may be of a compensatory nature, may have contributed to the present (or previously reported) effects.
}

negative information, it has been suggested that judgment of the valence of stimuli should be easier for positive than for negative stimuli, because the former ones form a more clearly identifiable category (i.e., density theory; Unkelbach et al., 2008; Unkelbach et al., 2010). However, such an account only applies to valence judgment tasks, but not to other tasks, such as speeded naming or lexical decision tasks, in which a speed advantage of positive over negative stimuli is found, as well (e.g., Kuperman et al., 2014). Furthermore, research has shown that a speed advantage of positive over negative responses even occurs when people respond with emotionally charged responses (i.e., approach and withdrawal) to stimuli they have no previous experience with (e.g., Chinese symbols; Kuhbandner et al., 2015), which additionally rules out recent extensions of the density theory that propose that differential subjective exposure frequencies may underlie the speed advantage of positive over negative responses (Unkelbach et al., 2010).

Another account is based on the mobilization-minimization hypothesis of Taylor (1991), which suggests that negative stimuli elicit a stronger mobilization of the organism than do positive stimuli, in that they elicit stronger physiological, emotional, and cognitive responses. According to some authors (e.g., Dijksterhuis \& Aarts, 2003), such a mobilization may explain the delayed responding to negative stimuli, because they seem to trigger more extensive and time-consuming cognitive analysis than do positive stimuli. However, a stronger "mobilization" of the organism can lead both to speeded and to delayed responding, depending on which subsystems of the organism are mobilized, which in turn should vary as a function of context. Imagine that a tiger surprises you by jumping out of the bush. In such a situation, an extensive cognitive analysis seems not to be very adaptive. Instead, energy should be mobilized to allow responding with appropriate behavior as quickly as possible. By contrast, if there is no time pressure for responding, an extensive analysis of negative stimuli is indeed adaptive, because this allows for developing more elaborated strategies for responding. Thus, if there is no time pressure for responding, a more extensive cognitive analysis may indeed underlie the slowed responding to negative stimuli. However, if a situation requires quick responding, as was the case in our study, where participants were instructed to respond as quickly as possible, it seems difficult to explain the slowed responding to negative stimuli by more extensive cognitive analysis. Given that we did not collect data on context effects and the amount of cognitive analysis, however, examining such effects would be an interesting avenue for future research.

To be able to examine the effects of emotional valence on sensory and response lateralization in oscillatory activity, stimuli have to be presented on either the left or the right, and participants have to respond to one emotional category with one hand and to the other with the other hand. Since it 
does not make sense to assign a third response category to one of the hands, we did not include emotionally neutral stimuli. Accordingly, the present study does not allow for determining the effects of positive and negative valence relative to a neutral condition. Thus, future research comparing both negative and positive with neutral stimuli will be needed to clarify whether the observed effects reflect mainly to effects of negative valence, positive valence, or both.

In conclusion, our findings have uncovered an unknown asymmetry between the processing of negative and positive stimuli at the level of cortical activations. Whereas negative stimuli are processed more efficiently than positive stimuli in perceptual networks, negative stimuli are processed less efficiently than positive stimuli in motor networks. Such an asymmetry may underlie the still unexplained discrepancy between perception and behavior when reacting to negative and positive stimuli, suggesting that although negative stimuli are actually quickly perceived, this advantage may not be reflected in response speeds due to a delay introduced by an increased variety of activated motor representations.

\section{References}

Anderson, J. R. (1974). Retrieval of propositional information from longterm memory. Cognitive Psychology, 6, 451-474.

Balota, D. A., \& Yap, M. J. (2011). Moving beyond the mean in studies of mental chronometry: The power of response time distributional analyses. Current Directions in Psychological Science, 20, 160166. doi:10.1177/0963721411408885

Baumeister, R. F., Bratslavsky, E., Finkenauer, C., \& Vohs, K. D. (2001). $\mathrm{Bad}$ is stronger than good. Review of General Psychology, 5, 323370. doi:10.1037/1089-2680.5.4.323

Bland, J. M., \& Altman, D. G. (1995). Calculating correlation coefficients with repeated observations: Part $1-$ Correlation within subjects. British Medical Journal, 310, 446.

Borgomaneri, S., Gazzola, V., \& Avenanti, A. (2014). Temporal dynamics of motor cortex excitability during perception of natural emotional scenes. Social Cognitive and Affective Neuroscience, 9, 14511457. doi:10.1093/scan/nst139

Brendl, C. M., Markman, A. B., \& Messner, C. (2005). Indirectly measuring evaluations of several attitude objects in relation to a neutral reference point. Journal of Experimental Social Psychology, 41, $346-368$.

Carretié, L., Albert, J., López-Martín, S., \& Tapia, M. (2009). Negative brain: An integrative review on the neural processes activated by unpleasant stimuli. International Journal of Psychophysiology, 71, $57-63$.

Christianson, S., \& Loftus, E. F. (1991). Remembering emotional events: The fate of detailed information. Cognition and Emotion, 5, 81-108. doi:10.1080/02699939108411027

Clark, H. H. (1973). The language-as-fixed-effect fallacy: A critique of language statistics in psychological research. Journal of Verbal Learning and Verbal Behavior, 12, 335-359. doi:10.1016/S00225371(73)80014-3

Cousineau, D. (2005). Confidence intervals in within-subject designs: A simpler solution to Loftus and Masson's method. Tutorial in Quantitative Methods for Psychology, 1, 42-45.
De Cesarei, A., \& Codispoti, M. (2011). Affective modulation of the LPP and $\alpha$-ERD during picture viewing. Psychophysiology, 48, 13971404.

de Jong, R., Gladwin, T. E., \& 't Hart, B. M. (2006). Movement-related EEG indices of preparation in task switching and motor control. Brain Research, 1105, 73-82.

de Oliveira, L. A. S., Imbiriba, L. A., Russo, M. M., Nogueira-Campos, A. A., Rodrigues, E. D. C., Pereira, M. G., ... Vargas, C. D. (2012). Preparing to grasp emotionally laden stimuli. PLOS ONE, 7, e45235. doi:10.1371/journal.pone.0045235

Dijksterhuis, A., \& Aarts, H. (2003). On wildebeests and humans: The preferential detection of negative stimuli. Psychological Science, 14, $14-18$.

Doyle, L. M. F., Yarrow, K., \& Brown, P. (2005). Lateralization of eventrelated beta desynchronization in the EEG during pre-cued reaction time tasks. Clinical Neurophysiology, 116, 1879-1888.

Eder, A. B., Leuthold, H., Rothermund, K., \& Schweinberger, S. R. (2012). Automatic response activation in sequential affective priming: An ERP study. Social Cognitive and Affective Neuroscience, 7 , 436-445. doi:10.1093/scan/nsr033

Ergenoglu, T., Demiralp, T., Bayraktaroglu, Z., Ergen, M., Beydagi, H., \& Uresin, Y. (2004). Alpha rhythm of the EEG modulates visual detection performance in humans. Cognitive Brain Research, 20, 376-383. doi:10.1016/j.cogbrainres.2004.03.009

Estes, Z., \& Verges, M. (2008). Freeze or flee? Negative stimuli elicit selective responding. Cognition, 108, 557-565.

Fox, E., Russo, B., Bowles, R., \& Dutton, K. (2001). Do threatening stimuli draw or hold visual attention in subclinical anxiety? Journal of Experimental Psychology: General, 130, 681-700. doi: 10.1037/0096-3445.130.4.681

Gray, J. A. (1987). The psychology of fear and stress (2nd ed.). Cambridge: Cambridge University Press.

Green, J. J., \& McDonald, J. J. (2010). The role of temporal predictability in the anticipatory biasing of sensory cortex during visuospatial shifts of attention. Psychophysiology, 47, 1057-1065. doi:10.1111/ j.1469-8986.2010.01025.x

Hillyard, S. A., \& Anllo-Vento, L. (1998). Event-related brain potentials in the study of visual selective attention. Proceedings of the National Academy of Sciences, 95, 781-787.

Hoechstetter, K., Bornfleth, H., Weckesser, D., Ille, N., Berg, P., \& Scherg, M. (2004). BESA source coherence: A new method to study cortical oscillatory coupling. Brain Topography, 16, 233-238.

Ille, N., Berg, P., \& Scherg, M. (2002). Artifact correction of the ongoing EEG using spatial filters based on artifact and brain signal topographies. Journal of Clinical Neurophysiology, 19, 113-124.

Joundi, R. A., Jenkinson, N., Brittain, J. S., Aziz, T. Z., \& Brown, P. (2012). Driving oscillatory activity in the human cortex enhances motor performance. Current Biology, 22, 403-407. doi:10.1016/j. cub.2012.01.024

Kaiser, J., Birbaumer, N., \& Lutzenberger, W. (2001). Event-related beta desynchronization indicates timing of response selection in a delayed-response paradigm in humans. Neuroscience Letters, 312, 149-152.

Kanske, P., \& Kotz, S. A. (2010). Leipzig Affective Norms for German: A reliability study. Behavior Research Methods, 42, 987-991. doi: 10.3758/BRM.42.4.987

Kelly, S. P., Lalor, E. C., Reilly, R. B., \& Foxe, J. J. (2006). Increases in alpha oscillatory power reflect an active retinotopic mechanism for distracter suppression during sustained visuospatial attention. Journal of Neurophysiology, 95, 3844-3851.

Kissler, J., Herbert, C., Peyk, P., \& Junghöfer, M. (2007). Buzzwords: Early cortical responses to emotional words during reading. Psychological Science, 18, 475-480.

Kousta, S.-T., Vinson, D. P., \& Vigliocco, G. (2009). Emotion words, regardless of polarity, have a processing advantage over 
neutral words. Cognition, 112, 473-481. doi:10.1016/j. cognition.2009.06.007

Krieglmeyer, R., \& Deutsch, R. (2013). Approach does not equal approach: Angry facial expressions evoke approach only when it serves aggression. Social Psychological and Personality Science, 4, 607-614

Kuhbandner, C., Hanslmayr, S., Maier, M. A., Pekrun, R., Spitzer, B., Pastötter, B., \& Bäuml, K.-H. (2009). Effects of mood on the speed of conscious perception: Behavioral and electrophysiological evidence. Social Cognitive and Affective Neuroscience, 4, 286-293. doi:10.1093/scan/nsp010

Kuhbandner, C., \& Pekrun, R. (2013). Joint effects of emotion and color on memory. Emotion, 13, 375-379.

Kuhbandner, C., Spitzer, B., \& Pekrun, R. (2011). Read-out of emotional information from iconic memory: The longevity of threatening stimuli. Psychological Science, 22, 695-700. doi:10.1177/ 0956797611406445

Kuhbandner, C., Vogel, C. M., \& Lichtenfeld, S. (2015). Switching from approach to withdrawal is easier than vice versa. Cognition and Emotion, 29, 1168-1184. doi:10.1080/02699931.2014.969197

Kuperman, V., Estes, Z., Brysbaert, M., \& Warriner, A. B. (2014). Emotion and language: Valence and arousal affect word recognition. Journal of Experimental Psychology: General, 143, 1065-1081.

Lahl, O., Göritz, A. S., Pietrowsky, R., \& Rosenberg, J. (2009). Using the World-Wide Web to obtain large-scale word norms: 190,212 ratings on a set of 2,654 German nouns. Behavior Research Methods, 41, 13-19. doi:10.3758/BRM.41.1.13

Larsen, R. J., Mercer, K. A., \& Balota, D. A. (2006). Lexical characteristics of words used in emotional Stroop experiments. Emotion, 6, 62-72. doi:10.1037/1528-3542.6.1.62

Lepaennen, J. M., \& Hietanen, J. K. (2004). Positive facial expressions are recognized faster than negative facial expressions, but why? Psychological Research, 69, 22-29.

Leuthold, H., Sommer, W., \& Ulrich, R. (1996). Partial advance information and response preparation: Inferences from the lateralized readiness potential. Journal of Experimental Psychology: General, 125, 307-323. doi:10.1037/0096-3445.125.3.307

Moller, A. C., Elliot, A. J., \& Maier, M. A. (2009). Basic hue-meaning associations. Emotion, 9, 898-902. doi:10.1037/a0017811

Nasrallah, M., Carmel, D., \& Lavie, N. (2009). "Murder she wrote": Enhanced sensitivity to negative word valence. Emotion, 9, 609618

Olivers, C. N. L., \& Nieuwenhuis, S. (2006). The beneficial effects of additional task load, positive affect, and instruction on the attentional blink. Journal of Experimental Psychology: Human Perception and Performance, 32, 364-379. doi:10.1037/0096-1523.32.2.364

Paulus, A., \& Wentura, D. (2016). It depends: Approach and avoidance reactions to emotional expressions are influenced by the contrast emotions presented in the task. Journal of Experimental
Psychology: Human Perception and Performance, 42, 197-212. doi:10.1037/xhp0000130

Pfurtscheller, G., \& Aranibar, A. (1977). Event-related cortical desynchronization detected by power measurements of scalp EEG. Electroencephalography and Clinical Neurophysiology, 42, 817-826.

Pfurtscheller, G., Brunner, C., Schlögl, A., \& Lopes da Silva, F. H. (1999). Mu rhythm (de)synchronization and EEG single-trial classification of different motor imagery tasks. NeuroImage, 31, 153-159.

Pogosyan, A., Gaynor, L. D., Eusebio, A., \& Brown, P. (2009). Boosting cortical activity at Beta-band frequencies slows movement in humans. Current Biology, 19, 1637-1641. doi:10.1016/j.cub.2009. 07.074

Ray, W. J., \& Cole, H. W. (1985). EEG alpha activity reflects attentional demands, and beta activity reflects emotional and cognitive processes. Science, 228, 750-752.

Simon, J. R. (1990). The effects of an irrelevant directional cue on human information processing. In R. W. Proctor \& T. G. Reeve (Eds.), Stimulus-response compatibility: An integrated perspective (pp. 31-86). Amsterdam: North-Holland.

Stancák, A., \& Pfurtscheller, G. (1996). Event-related desynchronisation of central beta-rhythms during brisk and slow self-paced finger movements of dominant and nondominant hand. Cognitive Brain Research, 4, 171-183.

Taylor, S. E. (1991). Asymmetrical effects of positive and negative events: The mobilization-minimization hypothesis. Psychological Review, 110, 67-85. doi:10.1037/0033-2909.110.1.67

Thut, G., Nietzel, A., Brandt, S. A., \& Pascual-Leone, A. (2006). Alphaband electro-encephalographic activity over occipital cortex indexes visuo-spatial attention bias and predicts visual target detection. Journal of Neuroscience, 26, 9494-9502.

Ulrich, R., \& Miller, J. (2001). Using the jackknife-based scoring method for measuring LRP onset effects in factorial designs. Psychophysiology, 38, 816-827.

Unkelbach, C., Fiedler, K., Bayer, M., Stegmüller, M., \& Danner, D. (2008). Why positive information is processed faster: The density hypothesis. Journal of Personality and Social Psychology, 95, 3649. doi:10.1037/0022-3514.95.1.36

Unkelbach, C., von Hippel, W., Forgas, J. P., Robinson, M. D., Shakarchi, R. J., \& Hawkins, C. (2010). Good things come easy: Subjective exposure frequency and the faster processing of positive information. Social Cognition, 28, 538-555.

Võ, M. L.-H., Conrad, M., Kuchinke, L., Urton, K., Hofmann, M. J., \& Jacobs, A. M. (2009). The Berlin Affective Word List Reloaded (BAWL-R). Behavior Research Methods, 41, 534-538. doi:10. 3758/BRM.41.2.534

Worden, M. S., Foxe, J. J., Wang, N., \& Simpson, G. V. (2000). Anticipatory biasing of visuospatial attention indexed by retinotopically specific alpha-band electroencephalography increases over occipital cortex. Journal of Neuroscience, 20, RC63. 